\title{
Locating-Total Dominating Sets in Twin-Free Graphs: a Conjecture
}

\author{
Florent Foucaud* Michael A. Henning \\ Department of Pure and Applied Mathematics \\ University of Johannesburg \\ Auckland Park, 2006, South Africa \\ florent.foucaud@gmail.com, mahenning@uj.ac.za
}

Submitted: Mar 30, 2015; Accepted: Jul 7, 2016; Published: Jul 22, 2016

Mathematics Subject Classifications: 05C69

\begin{abstract}
A total dominating set of a graph $G$ is a set $D$ of vertices of $G$ such that every vertex of $G$ has a neighbor in $D$. A locating-total dominating set of $G$ is a total dominating set $D$ of $G$ with the additional property that every two distinct vertices outside $D$ have distinct neighbors in $D$; that is, for distinct vertices $u$ and $v$ outside $D, N(u) \cap D \neq N(v) \cap D$ where $N(u)$ denotes the open neighborhood of $u$. A graph is twin-free if every two distinct vertices have distinct open and closed neighborhoods. The location-total domination number of $G$, denoted $\gamma_{t}^{L}(G)$, is the minimum cardinality of a locating-total dominating set in $G$. It is well-known that every connected graph of order $n \geqslant 3$ has a total dominating set of size at most $\frac{2}{3} n$. We conjecture that if $G$ is a twin-free graph of order $n$ with no isolated vertex, then $\gamma_{t}^{L}(G) \leqslant \frac{2}{3} n$. We prove the conjecture for graphs without 4-cycles as a subgraph. We also prove that if $G$ is a twin-free graph of order $n$, then $\gamma_{t}^{L}(G) \leqslant \frac{3}{4} n$.
\end{abstract}

Keywords: Locating-dominating sets; Total dominating sets; Dominating sets.

\section{Introduction}

A dominating set in a graph $G$ is a set $D$ of vertices of $G$ such that every vertex outside $D$ is adjacent to a vertex in $D$. The domination number, $\gamma(G)$, of $G$ is the minimum cardinality of a dominating set in $G$. A total dominating set, abbreviated TD-set, of $G$ is a set $D$ of vertices of $G$ such that every vertex of $G$ is adjacent to a vertex in $D$. The total domination number of $G$, denoted by $\gamma_{t}(G)$, is the minimum cardinality of a TD-set

* Part of this research was done while this author was a postdoctoral researcher at LIMOS, Université Blaise Pascal, Clermont-Ferrand, France. 
in $G$. The literature on the subject of domination parameters in graphs up to the year 1997 has been surveyed and detailed in the two books [14, 15], and a recent book on total dominating sets is also available [21].

Among the existing variations of (total) domination, the ones of location-domination and location-total domination are widely studied. A set $D$ of vertices locates a vertex $v$ if the neighborhood of $v$ within $D$ is unique among all vertices in $V(G) \backslash D$. A locatingdominating set is a dominating set $D$ that locates all the vertices, and the locationdomination number of $G$, denoted $\gamma_{L}(G)$, is the minimum cardinality of a locatingdominating set in $G$. A locating-total dominating set, abbreviated LTD-set, is a TDset $D$ that locates all the vertices, and the location-total domination number of $G$, denoted $\gamma_{t}^{L}(G)$, is the minimum cardinality of a LTD-set in $G$. The concept of a locatingdominating set was introduced and first studied by Slater [24, 25] (see also [9, 10, 12, 23, $26]$ ), and the additional condition that the locating-dominating set be a total dominating set was first considered in [16] (see also [1, 2, 3, 5, 6, 7, 18, 19]).

We remark that there are (twin-free) graphs with total domination number two and arbitrarily large location-total domination number. For $k \geqslant 3$, let $G_{k}$ be the graph obtained from $K_{2, k}$ as follows: select one of the two vertices of degree $k$ and subdivide every edge incident with it; then, add an edge joining the two vertices of degree $k$; finally, add two new vertices of degree 1 , each adjacent to one of the degree $k$-vertices. The resulting graph, $G_{k}$, has order $2 k+4$, total domination number 2 , and we claim that its location-total domination number is exactly one-half the order (namely, $k+2$ ). One possible LTD-set of $G_{k}$ consists of the two vertices of degree $k+1$, and for each pair of adjacent vertices of degree 2 , one of the vertices of that pair belongs to the LTD-set. The graph $G_{4}$, for example, is illustrated in Figure 1, where the darkened vertices form an LTD-set in $G_{4}$. To see that no smaller LTD-set exists, observe first that the two vertices of degree $k+1$ must belong to any LTD-set of $G_{k}$ (otherwise, the two vertices of degree 1 are not totally dominated). Moreover, consider any set of two pairs of adjacent vertices of degree 2 in $G_{k}$. In order for these four vertices to be located, at least one of them must belong to any LTD-set (otherwise, the ones adjacent to the same vertex of degree $k+1$ are not located). This shows that for at least $k-1$ pairs of adjacent degree 2-vertices, one member of that pair belongs to any LTD-set of $G_{k}$. Thus, any LTD-set of $G_{k}$ has size at least $k+1$. Assuming that we have an LTD-set of size exactly $k+1$, then we have a pair of adjacent vertices of degree 2 not belonging to the LTD-set, and moreover none of the degree 1-vertices belongs to the LTD-set. But then each of the two above degree 2-vertices and each degree 1-vertex of $G_{k}$ is totally dominated only by its neighbor of degree $k+1$ and is therefore not located, a contradiction. Hence $\gamma_{t}^{L}\left(G_{k}\right)=k+2$, as claimed.

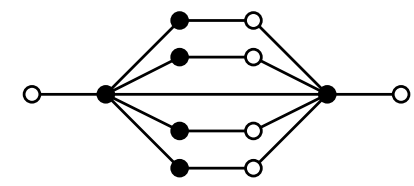

Figure 1: The twin-free graph $G_{4}$. 
A classic result due to Cockayne et al. [8] states that every connected graph of order at least 3 has a TD-set of cardinality at most two-thirds its order. While there are many graphs (without isolated vertices) which have location-total domination number much larger than two-thirds their order, the only such graphs that are known contain many twins, that is, pairs of vertices with the same closed or open neighborhood. We conjecture that in fact, twin-free graphs have location-total domination number at most two-thirds their order. In this paper we initiate the study of this conjecture.

Definitions and notations. For notation and graph theory terminology, we in general follow [14]. Specifically, let $G$ be a graph with vertex set $V(G)$, edge set $E(G)$ and with no isolated vertex. The open neighborhood of a vertex $v \in V(G)$ is $N_{G}(v)=\{u \in V \mid u v \in$ $E(G)\}$ and its closed neighborhood is the set $N_{G}[v]=N_{G}(v) \cup\{v\}$. The degree of $v$ is $d_{G}(v)=\left|N_{G}(v)\right|$. For a set $S \subseteq V(G)$, its open neighborhood is the set $N_{G}(S)=$ $\bigcup_{v \in S} N_{G}(v)$, and its closed neighborhood is the set $N_{G}[S]=N_{G}(S) \cup S$. Given a set $S \subset V(G)$ and a vertex $v \in S$, an $S$-external private neighbor of $v$ is a vertex outside $S$ that is adjacent to $v$ but to no other vertex of $S$ in $G$. The set of all $S$-external private neighbors of $v$, abbreviated $\operatorname{epn}_{G}(v, S)$, is the $S$-external private neighborhood. The subgraph induced by a set $S$ of vertices in $G$ is denoted by $G[S]$. If the graph $G$ is clear from the context, we simply write $V, E, N(v), N[v], N(S), N[S], d(v)$ and epn $(v, S)$ rather than $V(G), E(G), N_{G}(v), N_{G}[v], N_{G}(S), N_{G}[S], d_{G}(v)$ and $\operatorname{epn}_{G}(v, S)$, respectively.

Given a set $S$ of edges in $G$, we will denote by $G-S$ the subgraph obtained from $G$ by deleting all edges of $S$. For a set $S$ of vertices, $G-S$ is the graph obtained from $G$ by removing all vertices of $S$ and removing all edges incident to vertices of $S$. A cycle on $n$ vertices is denoted by $C_{n}$ and a path on $n$ vertices by $P_{n}$. The girth of $G$ is the length of a shortest cycle in $G$.

A set $D$ is a dominating set of $G$ if $N[v] \cap D \neq \varnothing$ for every vertex $v$ in $G$, or, equivalently, $N[D]=V(G)$. A set $D$ is a TD-set of $G$ if $N(v) \cap D \neq \varnothing$ for every vertex $v$ in $G$, or, equivalently, $N(D)=V(G)$. Two distinct vertices $u$ and $v$ in $V(G) \backslash D$ are located by $D$ if they have distinct neighbors in $D$; that is, $N(u) \cap D \neq N(v) \cap D$. If a vertex $u \in V(G) \backslash D$ is located from every other vertex in $V(G) \backslash D$, we simply say that $u$ is located by $D$.

A set $S$ is a locating set of $G$ if every two distinct vertices outside $S$ are located by $S$. In particular, if $S$ is both a dominating set and a locating set, then $S$ is a locating-dominating set. Further, if $S$ is both a TD-set and a locating set, then $S$ is a locating-total dominating set. We remark that the only difference between a locating set and a locating-dominating set in $G$ is that a locating set might have a unique non-dominated vertex.

Two distinct vertices $u$ and $v$ of a graph $G$ are open twins if $N(u)=N(v)$ and closed twins if $N[u]=N[v]$. Further, $u$ and $v$ are twins in $G$ if they are open twins or closed twins in $G$. A graph is twin-free if it has no twins.

For two vertices $u$ and $v$ in a connected graph $G$, the distance $d_{G}(u, v)$ between $u$ and $v$ is the length of a shortest $(u, v)$-path in $G$. The maximum distance among all pairs of vertices of $G$ is the diameter of $G$, which is denoted by $\operatorname{diam}(G)$. A nontrivial connected graph is a connected graph of order at least 2. A leaf of graph $G$ is a vertex of degree 1 , while a support vertex of $G$ is a vertex adjacent to a leaf. 
A rooted tree $T$ distinguishes one vertex $r$ called the root. For each vertex $v \neq r$ of $T$, the parent of $v$ is the neighbor of $v$ on the unique $(r, v)$-path, while a child of $v$ is any other neighbor of $v$. A descendant of $v$ is a vertex $u \neq v$ such that the unique $(r, u)$-path contains $v$. Thus, every child of $v$ is a descendant of $v$. We let $D(v)$ denote the set of descendants of $v$, and we define $D[v]=D(v) \cup\{v\}$. The maximal subtree at $v$ is the subtree of $T$ induced by $D[v]$, and is denoted by $T_{v}$.

The 2-corona of a graph $H$ is the graph of order $3|V(H)|$ obtained from $H$ by adding a vertex-disjoint copy of a path $P_{2}$ for each vertex $v$ of $H$ and adding an edge joining $v$ to one end of the added path.

We use the standard notation $[k]=\{1,2, \ldots, k\}$. If $A$ and $B$ are sets, then $A \times B=$ $\{(a, b) \mid a \in A, b \in B\}$.

Conjectures and known results. As a motivation for our study, we pose and state the following conjecture.

Conjecture 1. Every twin-free graph $G$ of order $n$ without isolated vertices satisfies $\gamma_{t}^{L}(G) \leqslant \frac{2}{3} n$.

In an earlier paper, Henning and Löwenstein [18] proved that every connected cubic claw-free graph (not necessarily twin-free) has a LTD-set of size at most one-half its order, which implies that Conjecture 1 is true for such graphs. Moreover they conjectured this to be true for every connected cubic graph, with two exceptions - which, if true, would imply Conjecture 1 for all cubic graphs.

A similar conjecture for locating-dominating sets, that motivated the present study, was posed in [13], and was strengthened in [12]. ${ }^{1}$

Conjecture 2 (Garijo, González, Márquez [13]). There exists an integer $n_{1}$ such that for any $n \geqslant n_{1}$, the maximum value of the location-domination number of a connected twin-free graph of order $n$ is $\left\lfloor\frac{n}{2}\right\rfloor$.

Conjecture 3 (Foucaud, Henning, Löwenstein, Sasse [11, 12]). Every twin-free graph $G$ of order $n$ without isolated vertices satisfies $\gamma_{L}(G) \leqslant \frac{n}{2}$.

Conjecture 3 remains open, although it was proved for a number of graph classes such as bipartite graphs and graphs with no 4-cycles [13], split and co-bipartite graphs [12], and cubic graphs [11]. Some of these results were obtained using selected vertex covers and matchings, but none of these techniques seems to be useful in the study of Conjecture 1 .

Our results. We prove the bound $\gamma_{t}^{L}(G) \leqslant \frac{3}{4} n$ in Section 3. We then give support to Conjecture 1 by proving it for graphs without 4-cycles in Section 4, where we also characterize all extremal examples without 4-cycles. (In this paper, by "graph with no 4-cycles", we mean that the graph does not contain any 4-cycle as a subgraph, whether the 4-cycle is induced or not.) We also discuss Conjecture 1 in relation with the minimum degree in Section 5, and we conclude the paper in Section 6.

\footnotetext{
${ }^{1}$ Note that in [12], we mistakenly attributed Conjecture 3 to the authors of [13]. We discuss this in more detail in [11].
} 


\section{Preliminaries}

This section contains a number of preliminary results that will be useful in the next sections.

Theorem 4 (Cockayne et al. [8]; Brigham et al. [4]). If $G$ is a connected graph of order $n \geqslant 3$, then $\gamma_{t}(G) \leqslant \frac{2}{3} n$. Further, $\gamma_{t}(G)=\frac{2}{3} n$ if and only if $G$ is isomorphic to a 3-cycle, a 6-cycle, or the 2-corona of some connected graph $H$.

We will need the following property of minimum TD-sets in a graph established in [17].

Theorem 5 ([17]). If $G$ is a connected graph of order $n \geqslant 3$, and $G \neq K_{n}$, then $G$ has a minimum $T D$-set $S$ such that every vertex $v \in S$ satisfies $|\operatorname{epn}(v, S)| \geqslant 1$ or has a neighbor $x$ in $S$ of degree 1 in $G[S]$ satisfying $|\operatorname{epn}(x, S)| \geqslant 1$.

Given a graph $G$, the set $L \cup T$, where $L$ is a locating-dominating set of $G$, and $T$ is a TD-set of $G$ is both a TD-set and a locating set, implying the following observation.

Observation 6. For every graph $G$ without isolated vertices, we have

$$
\gamma_{t}^{L}(G) \leqslant \gamma_{L}(G)+\gamma_{t}(G)
$$

\section{A general upper bound of three-quarters the order}

In this section we prove a general upper bound on the location-total domination number of a graph in terms of its order. The proof is similar to the bound $\gamma_{L}(G) \leqslant \frac{2}{3} n$ proved for locating-dominating sets in [12].

Theorem 7. If $G$ is a twin-free graph of order $n$ without isolated vertices, then $\gamma_{t}^{L}(G) \leqslant$ $\frac{3}{4} n$.

Proof. By linearity, we may assume that $G$ is connected. By the twin-freeness of $G$, we note that $n \geqslant 4$ and that $G \neq K_{n}$. For an arbitrary subset $S$ of vertices in $G$, let $\mathcal{P}_{S}$ be a partition of $\bar{S}=V(G) \backslash S$ with the property that all vertices in the same part of the partition have the same open neighborhood in $S$ and vertices from different parts of the partition have different open neighborhood in $S$. Let $\left|\mathcal{P}_{S}\right|=k(S)$. Let $X_{S}$ be the set of vertices in $\bar{S}$ that belong to a partition set in $\mathcal{P}_{S}$ of size 1 and let $Y_{S}=\bar{S} \backslash X_{S}$. Hence every vertex in $Y_{S}$ belongs to a partition set of size at least 2. Let $n_{1}(S)=\left|X_{S}\right|$ and let $n_{2}(S)=k(S)-n_{1}(S)$. Let $S$ be a minimum TD-set in $G$ with the property that every vertex $v \in S$ satisfies $|\operatorname{epn}(v, S)| \geqslant 1$ or has a neighbor $v^{\prime}$ in $S$ of degree 1 in $G[S]$ satisfying $\left|\operatorname{epn}\left(v^{\prime}, S\right)\right| \geqslant 1$. Such a set exists by Theorem 5 . We note that at least half the vertices in $S$ have an $S$-external private neighbor, implying that $n_{1}(S)+n_{2}(S) \geqslant \frac{1}{2}|S|$. Among all supersets $S^{\prime}$ of $S$ with the property that $n_{1}\left(S^{\prime}\right)+n_{2}\left(S^{\prime}\right) \geqslant \frac{1}{2}\left|S^{\prime}\right|$, let $D$ be chosen to be inclusion-wise maximal. (Possibly, $D=S$.)

Claim 7.A. The vertices in each partition set of size at least 2 in $\mathcal{P}_{D}$ have distinct neighborhoods in $X_{D}$, and $D \cup X_{D}$ is a LTD-set of $G$. 
Proof of Claim 7.A. Let $u$ and $v$ be two vertices that belong to a partition set $T$, of size at least 2 in $\mathcal{P}_{D}$. Since $G$ is twin-free, there exists a vertex $w \notin\{u, v\}$ that is adjacent to exactly one of $u$ and $v$. Since $u$ and $v$ have the same neighbors in $D$, we note that $w \notin D$. Hence, $w \in \bar{D}=V(G) \backslash D$. Suppose that $w \in Y_{D}$ and consider the set $D^{\prime}=D \cup\{w\}$. Let $R$ be an arbitrary partition set in $\mathcal{P}_{D}$ that might or might not contain $w$. If $w$ is either adjacent to every vertex of $R \backslash\{w\}$ or adjacent to no vertex in $R \backslash\{w\}$, then $R \backslash\{w\}$ is a partition set in $\mathcal{P}_{D^{\prime}}$. If $w$ is adjacent to some, but not all, vertices of $R \backslash\{w\}$, then there is a partition $R \backslash\{w\}=\left(R_{1}, R_{2}\right)$ of $R \backslash\{w\}$ where $R_{1}$ are the vertices in $R \backslash\{w\}$ adjacent to $w$ and $R_{2}$ are the remaining vertices in $R \backslash\{w\}$. In this case, both sets $R_{1}$ and $R_{2}$ form a partition set in $\mathcal{P}_{D^{\prime}}$. In particular, we note that there is a partition $T \backslash\{w\}=\left(T_{1}, T_{2}\right)$ of $T \backslash\{w\}$ where both sets $T_{1}$ and $T_{2}$ form a partition set in $\mathcal{P}_{D^{\prime}}$. Therefore, $n_{1}\left(D^{\prime}\right)+n_{2}\left(D^{\prime}\right) \geqslant n_{1}(D)+n_{2}(D)+1 \geqslant \frac{1}{2}|D|+1>\frac{1}{2}(|D|+1)=\frac{1}{2}\left|D^{\prime}\right|$, contradicting the maximality of $D$. Hence, $w \notin Y_{D}$. Therefore, $w \in X_{D}$. Hence, $u$ and $v$ are located by the set $X_{D}$ in $G$. Moreover, $D \cup X_{D}$ is a TD-set since $D$ itself is a TD-set. (口)

Let $Y_{D}^{\prime}$ be obtained from $Y_{D}$ by deleting one vertex from each partition set of size at least 2 in $\mathcal{P}_{D}$, and let $D^{\prime}=D \cup Y_{D}^{\prime}$. Then, $\left|D^{\prime}\right|=n-n_{1}(D)-n_{2}(D)$. By definition of the partition $\mathcal{P}_{D}$, every vertex in $V(G) \backslash D^{\prime}$ has a distinct nonempty neighborhood in $D$ and therefore in $D^{\prime}$. Moreover, $D^{\prime}$ is a TD-set since $D$ itself is a TD-set. Hence we have the following claim.

Claim 7.B. The set $D^{\prime}$ is a LTD-set of $G$.

Let $n_{1}=n_{1}(D)$ and $n_{2}=n_{2}(D)$. By Claim 7.A, the set $D \cup X_{D}$ is a LTD-set of $G$ of cardinality $|D|+n_{1}$. By Claim 7.B, the set $D^{\prime}$ is a LTD-set of $G$ of cardinality $n-n_{1}-n_{2}$. Hence,

$$
\gamma_{t}^{L}(G) \leqslant \min \left\{|D|+n_{1}, n-n_{1}-n_{2}\right\} .
$$

Inequality (1) implies that if $n-n_{1}-n_{2} \leqslant \frac{3}{4} n$, then $\gamma_{L}(G) \leqslant \frac{3}{4} n$. Hence we may assume that $n-n_{1}-n_{2}>\frac{3}{4} n$, for otherwise the desired upper bound on $\gamma_{t}^{L}(G)$ follows. With this assumption, $n_{1}+n_{2}<\frac{1}{4} n$. By our choice of the set $D$, we recall that $|D| \leqslant 2\left(n_{1}+n_{2}\right)$. Therefore,

$$
|D|+n_{1} \leqslant 3 n_{1}+2 n_{2} \leqslant 3\left(n_{1}+n_{2}\right)<\frac{3}{4} n
$$

Hence, by Inequality $(1), \gamma_{t}^{L}(G)<\frac{3}{4} n$. This completes the proof of Theorem 7 .

\section{Graphs without 4-cycles}

In this section, we prove Conjecture 1 for graphs with no 4-cycles. We also characterize all graphs with no 4-cycles that achieve the bound of Conjecture 1. Surprisingly, these are precisely those graphs that have no 4-cycles and no twins and that are extremal for the bound on the total domination number from Theorem 4. This is in stark contrast with Conjecture 3 for the location-domination number, where many graphs (without 4-cycles) 
are known that are extremal for the conjecture but have much smaller domination number than one-half the order, see [12].

Theorem 8. Let $G$ be a twin-free graph of order $n$ without isolated vertices and 4-cycles. Then, $\gamma_{t}^{L}(G) \leqslant \frac{2}{3} n$. Further, $\gamma_{t}^{L}(G)=\frac{2}{3} n$ if and only if $G$ is isomorphic to a 6-cycle or is the 2-corona of some connected nontrivial graph that contains no 4-cycles.

Proof. We prove the theorem by induction on $n$. By linearity, we may assume that $G$ is connected, for otherwise we apply induction to each component of $G$ and we are done. By the twin-freeness of $G$, we note that $n \geqslant 4$. Further if $n=4$, then since $G$ is $C_{4}$-free, the graph $G$ is the path $P_{4}$ and $\gamma_{t}^{L}\left(P_{4}\right)=2<\frac{2}{3} n$. This establishes the base case. Let $n \geqslant 5$ and assume that every twin-free graph $G^{\prime}$ without isolated vertices and with no 4-cycles of order $n^{\prime}$, where $n^{\prime}<n$, satisfies $\gamma_{t}^{L}\left(G^{\prime}\right) \leqslant \frac{2}{3} n^{\prime}$, and that the only graphs achieving the bound are the extremal graphs described in Theorem 4 that are twin-free and have no 4-cycles. Let $G$ be a twin-free graph without isolated vertices and with no 4-cycles of order $n$. The general idea will be to partition $V(G)$ into two sets $V_{1}$ and $V_{2}$. If $G\left[V_{1}\right]$ and/or $G\left[V_{2}\right]$ are twin-free, we apply induction, and use the obtained LTD-sets of $G\left[V_{1}\right]$ and/or $G\left[V_{2}\right]$ to build one of $G$. We proceed further with the following series of claims.

Claim 8.A. If $G$ is a tree, then $\gamma_{t}^{L}(G) \leqslant \frac{2}{3} n$. Further, $\gamma_{t}^{L}(G)=\frac{2}{3} n$ if and only if $G$ is the 2-corona of a nontrivial tree.

Proof of Claim 8.A. Suppose that $G$ is a tree. Since $n \geqslant 5$, we note that $\operatorname{diam}(G) \geqslant 4$ (otherwise $G$ contains twin vertices of degree 1). For the same reason, if $\operatorname{diam}(G)=4$, then either $G=P_{5}$ or $G$ is obtained from a star $K_{1, k+1}$, where $k \geqslant 2$, by subdividing at least $k$ edges of the star exactly once. In this case, the set of vertices of degree at least 2 in $G$ forms a LTD-set of size strictly less than two-thirds the order. Hence, we may assume that $\operatorname{diam}(G) \geqslant 5$, for otherwise the desired result follows.

Let $P$ be a longest path in $G$ and let $P$ be an $(r, u)$-path. Necessarily, both $r$ and $u$ are leaves. Since $\operatorname{diam}(G) \geqslant 5$, we note that $P$ has length at least 5 . We now root the tree at the vertex $r$. Let $v$ be the parent of $u$, and let $w$ be the parent of $v, x$ the parent of $w$, and $y$ the parent of $x$ in the rooted tree. Since $|V(P)| \geqslant 6$, we note that $y \neq r$. Since $G$ is twin-free, the vertex $w$ has at most one leaf-neighbor and every child of $w$ that is not a leaf has degree $2 \mathrm{in} G$. In particular, $d_{G}(v)=2$. We now consider the subtree $G_{w}$ of $G$ rooted at the vertex $w$. If $d_{G}(w)=2$, then $G_{w}=P_{3}$, while if $d_{G}(w) \geqslant 3$, then $G_{w}$ is obtained from a star $K_{1, k+1}$, where $k \geqslant 1$, by subdividing at least $k$ edges of the star exactly once. Let $G^{\prime}=G-V\left(G_{w}\right)$.

We now define the subtrees $G_{1}$ and $G_{2}$ of $G$ as follows. We distinguish two cases; in both of them, $G_{2}$ is twin-free.

- If the tree $G^{\prime}$ is twin-free, then we let $V_{1}=V\left(G_{w}\right)$ and $V_{2}=V(G) \backslash V_{1}$, and we let $G_{1}=G\left[V_{1}\right]$ and $G_{2}=G\left[V_{2}\right]$. We note that in this case, $G_{2}=G^{\prime}$.

- If the tree $G^{\prime}$ is not twin-free, then necessarily, the parent $x$ of $w$ has a twin $x^{\prime}$ in $G^{\prime}$, and $N_{G}\left(x^{\prime}\right)=N_{G}(x) \backslash\{w\}=\{y\}$. Thus, $d_{G}(x)=2$ and the vertex $x^{\prime}$ is 
a leaf-neighbor of $y$ in $G$. Moreover, we claim that if $x^{\prime}=r$, then we are done. Indeed, in this case, our choice of $P$ as a longest path in $G$ implies that $G^{\prime}$ is the path ryx. If now $G_{w} \neq P_{3}$, then the set of vertices of degree at least 2 in $G$ forms a LTD-set of $G$ of size strictly less than two-thirds the order, while if $G_{w}=P_{3}$, then $G$ is the path $P_{6}$, which is the 2-corona of a tree $K_{2}$, and $\gamma_{t}^{L}(G)=\frac{2}{3} n$. In both cases we are done. Thus, we may assume that $x^{\prime} \neq r$. We now let $V_{1}=V\left(G_{w}\right) \cup\{x\}$, $V_{2}=V(G) \backslash V_{1}$, and we let $G_{1}=G\left[V_{1}\right]$ and $G_{2}=G\left[V_{2}\right]$. We note that in this case, $G_{2}=G^{\prime}-x$. Our assumption that $x^{\prime} \neq r$ implies that $G_{2}$ is a twin-free tree.

Let $D_{2}$ be a minimum LTD-set of $G_{2}$. Applying the induction hypothesis to the twinfree tree $G_{2}$, the set $D_{2}$ satisfies $\left|D_{2}\right| \leqslant \frac{2}{3}\left|V_{2}\right|$. Further, if $\left|D_{2}\right|=\frac{2}{3}\left|V_{2}\right|$, then $G_{2}$ is the 2 -corona of a nontrivial tree. Let $D_{1}$ consist of $w$ and every child of $w$ of degree 2. Then, $\left|D_{1}\right| \leqslant \frac{2}{3}\left|V_{1}\right|$ with strict inequality if $G_{1}$ is not the path uvw. We claim that $D=D_{1} \cup D_{2}$ is a LTD-set of $G$. Since $D_{1}$ and $D_{2}$ are TD-sets of $G_{1}$ and $G_{2}$, respectively, the set $D$ is a TD-set of $G$. Every vertex of $G$ is located by $D$ except possibly for the vertex $x$ and a leaf-neighbor of $w$ in $G$, if such a leaf-neighbor exists. If $x \in V\left(G_{2}\right)$, then it is located in $G_{2}$ and hence in $G$. If $x \in V\left(G_{1}\right)$, then its twin $x^{\prime}$ in $G^{\prime}$ is a leaf-neighbor of $y$, implying that in $G_{2}$ the support vertex $y \in D_{2}$. Thus, $x$ is located by $w$ and $y$. If $w$ has a leaf-neighbor in $G$, then such a leaf-neighbor is located by $w$ only. Therefore, $D$ is a LTD-set of $G$, and so

$$
\gamma_{t}^{L}(G) \leqslant|D|=\left|D_{1}\right|+\left|D_{2}\right| \leqslant \frac{2}{3}\left|V_{1}\right|+\frac{2}{3}\left|V_{2}\right|=\frac{2}{3} n .
$$

This establishes the desired upper bound. Suppose next that $\gamma_{t}^{L}(G)=\frac{2}{3} n$. Then we must have equality throughout the Inequality Chain (2). In particular, $\left|D_{1}\right|=\frac{2}{3}\left|V_{1}\right|$ and $\left|D_{2}\right|=\frac{2}{3}\left|V_{2}\right|$, implying that $G_{1}=P_{3}$ (and $G_{1}$ consists of the path uvw) and $G_{2}$ is the 2-corona of a nontrivial tree, say $T_{2}$. Let $A$ and $B$ be the set of leaves and support vertices, respectively, in $G_{2}$, and let $C$ be the remaining vertices of $G_{2}$. We note that $C=V\left(T_{2}\right)=V_{2} \backslash(A \cup B)$ and $|C| \geqslant 2$ (since $T_{2}$ is a nontrivial tree). If $x \in A$, then $x$ is a leaf in $G_{2}$ and its neighbor $y$ is a support vertex in $G_{2}$ and belongs to the set $B$. If $x \in B$, then $x$ is a support vertex in $G_{2}$ and its parent $y$ belongs to $C$. In both cases, the set $(B \cup C \cup\{v, w\}) \backslash\{y\}$ is a LTD-set of $G$ of size $\left|D_{1}\right|+\left|D_{2}\right|-1=\frac{2}{3} n-1$, a contradiction to our supposition that $\gamma_{t}^{L}(G)=\frac{2}{3} n$. Hence, $x \in C$, implying that $G$ is the 2-corona of a nontrivial tree, namely the tree $G[C \cup\{w\}]$ obtained from $T_{2}$ by adding to it the vertex $w$ and the edge $w x$. This completes the proof of Claim 8.A. (口)

By Claim 8.A, we may assume that $G$ is not a tree, for otherwise the desired result follows. Hence, $G$ contains a cycle. We consider next the case when $G$ contains a triangle.

Claim 8.B. If $G$ contains a triangle, then $\gamma_{t}^{L}(G) \leqslant \frac{2}{3} n$. Further, $\gamma_{t}^{L}(G)=\frac{2}{3} n$ if and only if $G$ is isomorphic to a 6 -cycle or is the 2-corona of some connected nontrivial graph that contains no 4-cycles but contains a triangle.

Proof of Claim 8.B. Suppose that $G$ contains a triangle $C$. Let $G^{\prime}=G-V(C)$. We build a subset $V_{1}$ of vertices of $G$ as follows. Let $V_{0}$ consist of $V(C)$ together with all vertices 
that belong to a component $C^{\prime}$ of $G^{\prime}$ isomorphic to $P_{1}, P_{2}$ or $P_{3}$. We remark that if $C^{\prime}$ is a $P_{1^{-}}$or $P_{2}$-component of $G^{\prime}$, then at most one edge joins it to $C$, for otherwise there would be a 4-cycle or a pair of twins in $G$. Suppose that $S$ is a set of mutual twins of $G-V_{0}$. Since $G$ is twin-free, all but possibly one vertex in $S$ must be adjacent to a vertex of $C$. For each such set $S$ of mutual twins of $G-V_{0}$, we select $|S|-1$ vertices from $S$ that have a neighbor in $C$, and add these vertices to the set $V_{0}$ to form the set $V_{1}$ (possibly, $\left.V_{1}=V_{0}\right)$. Let $V_{2}=V(G) \backslash V_{1}$. Let $G_{1}=G\left[V_{1}\right]$ and if $V_{2} \neq \varnothing$, let $G_{2}=G\left[V_{2}\right]$. We note that $G_{1}$ is connected, while $G_{2}$ may possibly be disconnected.

Subclaim 8.B.1 $G_{2}$ is twin-free and has no isolated vertices.

Proof of Subclaim 8.B.1. We first prove that $G_{2}$ is twin-free. Suppose, to the contrary, that there is a pair $\left\{t, t^{\prime}\right\}$ of twins in $G_{2}$. By construction of $V_{2}$, the vertices $t$ and $t^{\prime}$ are not twins in $G-V_{0}$, implying that there exists a vertex $v$ in $V_{1} \backslash V_{0}$ such that $v$ is adjacent to exactly one of $t$ and $t^{\prime}$, say to $t$. Let $v^{\prime}$ be the twin of $v$ in $G-V_{0}$ that was not added to the set $V_{1}$ (recall that by construction, all but one vertex from a set of mutual twins in $G-V_{0}$ is added to the set $V_{1}$ ). But then, $v^{\prime}$ is a vertex in $G_{2}$ that is adjacent to $t$ but not to $t^{\prime}$, contradicting our supposition that $t$ and $t^{\prime}$ are twins in $G_{2}$. Therefore, $G_{2}$ is twin-free. The proof that $G_{2}$ has no isolated vertices, again by the construction, an isolated vertex $x$ would have been a neighbor of a set of twins of $G-V_{0}$. But at least one twin still belongs to $G_{2}$, and $x$ is not isolated. (口)

By Subclaim 8.B.1, $G_{2}$ is twin-free. Let $D_{2}$ be a minimum LTD-set of $G_{2}$. Applying the induction hypothesis to each component of $G_{2}$, the set $D_{2}$ satisfies $\left|D_{2}\right| \leqslant \frac{2}{3}\left|V_{2}\right|$. Further, if $\left|D_{2}\right|=\frac{2}{3}\left|V_{2}\right|$, then each component of $G_{2}$ is isomorphic to a 6-cycle or is the 2-corona of some connected nontrivial graph that contains no 4-cycles.

We note that the graph $G_{1}$ could have twins. For example, this would occur if $V_{1}=$ $V(C)$, in which case $G_{1}$ is the 3-cycle $C$. A more complicated possibility is if there were twins $t$ and $t^{\prime}$ in $G-V_{0}$; then at least one of them belongs to $G_{1}$ and could be, in $G_{1}$, a twin with the vertex of some $P_{1}$-component of $G^{\prime}$. Let us build a set $D_{1} \subset V_{1}$. As observed earlier, if $C^{\prime}$ is a $P_{1^{-}}$or $P_{2}$-component of $G^{\prime}$, then at most one edge joins it to $C$. For every $P_{3}$-component $C^{\prime}$ of $G^{\prime}$, select the central vertex of $C^{\prime}$ and one of its neighbors in $C^{\prime}$ that is not a leaf in $G$ and add these two vertices of $C^{\prime}$ to $D_{1}$. For every $P_{2}$-component $C^{\prime}$ of $G^{\prime}$, add to $D_{1}$ the unique vertex of $C^{\prime}$ adjacent to a vertex of $C$, as well as its neighbor in $C$. For every $P_{1}$-component of $G^{\prime}$ consisting of a vertex $v^{\prime}$, add to $D_{1}$ the unique neighbor of $v^{\prime}$ in $C$. For every vertex in $V_{1} \backslash V_{0}$ that had a twin in $G-V_{0}$, add its neighbor in $C$ to $D_{1}$. Now, if there is at most one vertex of $C$ in the resulting set $D_{1}$, then we augment $D_{1}$ so that exactly two vertices of $C$ belong to $D_{1}$. By construction the resulting set $D_{1}$ is a TD-set of $G_{1}$ and $\left|D_{1}\right| \leqslant \frac{2}{3}\left|V_{1}\right|$.

Subclaim 8.B.2 $D=D_{1} \cup D_{2}$ is a LTD-set of $G$.

Proof of Subclaim 8.B.2. Since $D_{1}$ and $D_{2}$ are TD-sets of $G_{1}$ and $G_{2}$, respectively, the set $D$ is a TD-set of $G$. Suppose, to the contrary, that $D$ is not locating. Then there is a pair of vertices, $u$ and $v$, that is not located by $D$. If $(u, v) \in V_{1} \times V_{2}$ (that is, $u \in V_{1}$ 
and $v \in V_{2}$ ), then $u$ is dominated by a vertex of $D_{1}$ and $v$ is dominated by a vertex of $D_{2}$. Hence, $u$ and $v$ must both be dominated by these two vertices. But then we have a 4-cycle in $G$, a contradiction. Hence, $(u, v) \notin V_{1} \times V_{2}$. Analogously, $(u, v) \notin V_{2} \times V_{1}$. Since $D_{2}$ is locating in $G_{2}$, we note that $(u, v) \notin V_{2} \times V_{2}$. Hence, $(u, v) \in V_{1} \times V_{1}$; that is, both $u$ and $v$ belong to $G_{1}$. Moreover $u$ cannot belong to $C$, for otherwise $u$ is dominated by two vertices in $D_{1} \cap C$ and is located. Similarly, $v \notin C$. Analogously, $u$ and $v$ cannot belong to a $P_{1^{-}}, P_{2^{-}}$or $P_{3^{-}}$-component of $G^{\prime}$, for otherwise it would be the only vertex in $V(G) \backslash D$ that is dominated only by its unique neighbor in $D_{1}$. Therefore, both $u$ and $v$ belong to $V_{1} \backslash V_{0}$ and had a twin in $G-V_{0}$. Let $u^{\prime}$ be the twin of $u$ in $G-V_{0}$ that was not added to the set $V_{1}$, and so $u^{\prime} \in V_{2}$. If $u$ and $u^{\prime}$ are open twins in $G-V_{0}$, then $u^{\prime}$ is a vertex of degree 1 in $G$, for otherwise $u$ and $u^{\prime}$ belong to a 4-cycle. For the same reason, if $u$ and $u^{\prime}$ are closed twins, then $u^{\prime}$ has degree 2 in $G$. In both cases, $u^{\prime}$ has degree 1 in $G_{2}$. The unique common neighbor of $u$ and $u^{\prime}$ therefore belongs to $D_{2}$ in order to totally dominate the vertex $u^{\prime}$ in $G_{2}$. Thus, $u$ is dominated by a vertex of $D_{1}$ and a vertex of $D_{2}$. Since $u$ and $v$ are not located, $v$ is also dominated by these two vertices, which implies that $u$ and $v$ belong to a common 4-cycle of $G$, a contradiction. Therefore, $D$ is a LTD-set of $G$. (口)

By Subclaim 8.B.2, the set $D=D_{1} \cup D_{2}$ is a LTD-set of $G$, implying that the Inequality Chain (2) presented in the proof of Claim 8.A holds. This establishes the desired upper bound.

Suppose next that $\gamma_{t}^{L}(G)=\frac{2}{3} n$. Then we must have equality throughout the Inequality Chain (2). In particular, $\left|D_{1}\right|=\frac{2}{3}\left|V_{1}\right|$ and $\left|D_{2}\right|=\frac{2}{3}\left|V_{2}\right|$. Since $\left|D_{1}\right|=\frac{2}{3}\left|V_{1}\right|$, our construction of the set $D_{1}$ implies that no component of $G^{\prime}$ is isomorphic to $P_{1}$ and that $V_{1}=V_{0}$. Further, if $G^{\prime}$ contains a $P_{2}$-component, then it has exactly three $P_{2}$-components each being joined via exactly one edge to a distinct vertex of $C$. In addition, there may be some, including the possibility of none, $P_{3}$-components in $G^{\prime}$. Suppose that $P^{\prime}$ is a $P_{3}$-component in $G^{\prime}$ and $x$ is a vertex of $P^{\prime}$ that is adjacent to a vertex of $C$. Then, $x$ is a leaf of $P^{\prime}$ and is adjacent to exactly one vertex of $C$, since $G$ is twin-free and has no 4-cycles. Suppose, further, that both leaves of $P^{\prime}$ are adjacent to (distinct) vertices of $C$. Let $u$ and $v$ be two (distinct) vertices of $C$ joined to $P^{\prime}$. If exactly one of $u$ and $v$ belong to $D_{1}$, then by our earlier observations, $G^{\prime}$ contains no $P_{2}$-component. But then by the way in which the set $D_{1}$ is constructed and recalling that $G^{\prime}$ contains no $P_{1}$-component and that $V_{1}=V_{0}$, we would have chosen two arbitrary vertices of $C$ to add to $D_{1}$. Hence, we can replace the two vertices of $C$ that currently belong to $D_{1}$ with the two vertices $u$ and $v$. We may therefore assume that $D_{1}$ is chosen to contain both $u$ and $v$. With this assumption, we can replace the two vertices of $P^{\prime}$ that currently belong to $D_{1}$ with one of the leaves of $P^{\prime}$ to produce a new LTD-set of $G$ of size $|D|-1=\gamma_{t}^{L}(G)-1$, a contradiction. Therefore, $P^{\prime}$ is joined via exactly one edge to a vertex of $C$. Thus, there are two possible structures of the graph $G_{1}$, described as follows.

Structure 1. The graph $G_{1}$ is obtained from the 3-cycle $C$ by adding any number of vertex-disjoint copies of $P_{3}$, including the possibility of zero, and joining an end from each such added path to exactly one vertex of $C$. 
Structure 2. The graph $G_{1}$ is obtained from the 2-corona of the 3-cycle $C$ by adding any number of vertex-disjoint copies of $P_{3}$, including the possibility of zero, and joining an end from each such added path to exactly one vertex of $C$.

We note that if $G_{1}$ has the structure described in Structure 2, then $G_{1}$ is the 2-corona of some connected nontrivial graph, say $H_{1}$, that contains the triangle $C$ and contains no 4-cycles. Further we note that if $x \in V\left(H_{1}\right)$, then either $x \in V(C)$ or $x$ is the vertex of a $P_{3}$-component in $G^{\prime}$ that is adjacent to a vertex of $C$.

Subclaim 8.B.3 If $G=G_{1}$, then the graph $G$ is the 2-corona of some connected nontrivial graph that contains the triangle $C$ and contains no 4-cycles.

Proof of Subclaim 8.B.3. Suppose that $G=G_{1}$, i.e., $V_{2}=\varnothing$. We first show that $G$ has the structure described in Structure 2. Suppose to the contrary that $G$ has the structure described in Structure 1. Then, since $G$ is twin-free, the graph $G$ is obtained from the 3-cycle $C$ by adding $k \geqslant 2$ vertex-disjoint copies of $P_{3}$ and joining an end from each such added path to exactly one vertex of $C$. Further, by the twin-freeness of $G$, at least two vertices of $C$ are joined to an end of an added path. Let $u$ and $v$ be two (distinct) vertices of $C$ are joined to ends of added paths $P_{3}$. The set of $2 k$ vertices of degree 2 in $G$ that belong to added paths, together with the vertex $u$, forms a LTD-set of $G$ of size $\frac{2}{3} n-1$, a contradiction. Therefore, $G$ has the structure described in Structure 2. Thus, the graph $G$ is the 2-corona of some connected nontrivial graph that contains the triangle $C$ and contains no 4-cycles. (口)

By Subclaim 8.B.3, we may assume that $G \neq G_{1}$, for otherwise the desired result follows. Hence, $V_{2} \neq \varnothing$. Since $\left|D_{2}\right|=\frac{2}{3}\left|V_{2}\right|$, applying the inductive hypothesis to each component of $G_{2}$, we deduce that each component of $G_{2}$ is isomorphic to a 6-cycle or is the 2-corona of some connected nontrivial graph that contains no 4-cycles.

Subclaim 8.B.4 No component of $G_{2}$ is isomorphic to a 6 -cycle.

Proof of Subclaim 8.B.4. Suppose, to the contrary, that $G_{2}$ contains a component $C^{\prime}$ that is isomorphic to a 6 -cycle. Since $G$ is connected, there is an edge that joins a vertex $x \in V(C)$ and a vertex $y \in V\left(C^{\prime}\right)$. Let $C^{\prime}$ be given by $y_{1} y_{2} \ldots y_{6} y_{1}$, where $y=y_{1}$. If $G_{1}$ has the structure described in Structure 1, then we can choose $D_{1}$ to contain any two vertices of $C$. Hence we may assume that in this case, $D_{1}$ is chosen to contain the vertex $x$. If $G_{1}$ has the structure described in Structure 2, then $V(C) \subset D_{1}$. In particular, $x \in D_{1}$. Hence, in both cases, $x \in D_{1}$. Replacing the four vertices of $D$ that belong to the component $C^{\prime}$ with the three vertices $\left\{y_{3}, y_{4}, y_{5}\right\}$ produces a LTD-set of $G$ of size $|D|-1=\frac{2}{3} n-1$, a contradiction. (ㅁ)

By Subclaim 8.B.4, each component of $G_{2}$ is the 2-corona of some connected nontrivial graph that contains no 4-cycles, implying that the graph $G_{2}$ is the 2-corona of some graph, say $\mathrm{H}_{2}$, that contains no 4-cycles. Moreover, since $G_{2}$ is twin-free, each component of $\mathrm{H}_{2}$ is nontrivial. Let $A_{2}$ and $B_{2}$ be the set of leaves and support vertices, respectively, in $G_{2}$, and let $C_{2}$ be the remaining vertices of $G_{2}$. We note that $C_{2}=V\left(H_{2}\right)=V_{2} \backslash\left(A_{2} \cup B_{2}\right)$. 
Subclaim 8.B.5 $G_{1}$ has the structure described in Structure 2.

Proof of Subclaim 8.B.5. Suppose, to the contrary, that $G_{1}$ has the structure described in Structure 1. Then, the graph $G_{1}$ is obtained from the 3 -cycle $C$ by adding $k \geqslant 0$ vertex-disjoint copies of $P_{3}$ and joining an end from each such added path to exactly one vertex of $C$. Let $V(C)=\{u, v, w\}$. If at least two vertices of $C$ are joined to an end of an added path, then analogously as in the proof of Subclaim 8.B.3, we produce a LTD-set of $G$ of size $\frac{2}{3} n-1$, a contradiction. Hence, either $G_{1}=C_{3}$ or $G_{1}$ is obtained from the 3 -cycle $C$ by adding $k \geqslant 1$ vertex-disjoint copies of $P_{3}$ and joining an end from each such added path to the same vertex of $C$, say to $u$. In both cases, both $v$ and $w$ have degree 2 in $G_{1}$. Since $G$ is twin-free, at least one of $v$ and $w$, say $v$, is adjacent to a vertex of $V_{2}$. If $v$ is adjacent to a vertex of $A_{2} \cup B_{2}$, then an analogous argument as in the last paragraph of the proof of Claim 8.A produces a LTD-set of $G$ of size $\frac{2}{3} n-1$, a contradiction. Hence, the neighbors of $v$ in $V_{2}$ all belong to $C_{2}$. Analogously, the neighbors of $u$ and $w$ in $V_{2}$, if any exist, all belong to $C_{2}$. The set of $2 k$ vertices of degree 2 in $G$ that belong to the added $P_{3}$-paths in $G_{1}$, together with the set $B_{2} \cup C_{2} \cup\{v\}$, is a LTD-set of $G$ of size $\frac{2}{3} n-1$, a contradiction. (ㅁ)

By Subclaim 8.B.5, $G_{1}$ has the structure described in Structure 2, implying that $G_{1}$ is the 2-corona of some connected nontrivial graph, say $H_{1}$, that contains the triangle $C$ and contains no 4-cycles. Let $A_{1}$ and $B_{1}$ be the set of leaves and support vertices, respectively, in $G_{1}$, and let $C_{1}$ be the remaining vertices of $G_{1}$. We note that $C_{1}=$ $V\left(H_{1}\right)=V_{1} \backslash\left(A_{1} \cup B_{2}\right)$.

Since $G$ is connected, there is an edge in $G$ joining a vertex $x \in V_{1}$ and a vertex $y \in V_{2}$. Let $a_{1} b_{1} c_{1}$ be the path in $G_{1}$ containing $x$, where $a_{1} \in A_{1}, b_{1} \in B_{1}$ and $c_{1} \in C_{1}$. Similarly, let $a_{2} b_{2} c_{2}$ be the path in $G_{2}$ containing $y$, where $a_{2} \in A_{2}, b_{2} \in B_{2}$ and $c_{2} \in C_{2}$. We show that $x=c_{1}$. Suppose, to the contrary, that $x \in\left\{a_{1}, b_{1}\right\}$. Let $D^{*}=C_{1} \cup C_{2} \cup B_{1} \cup B_{2}$. If $x y=a_{1} a_{2}$, let $X=\left(D^{*} \cup\left\{a_{1}, a_{2}\right\}\right) \backslash\left\{b_{1}, b_{2}, c_{1}\right\}$. If $x y \in\left\{a_{1} b_{2}, a_{1} c_{2}\right\}$, let $X=\left(D^{*} \cup\left\{a_{1}\right\}\right) \backslash$ $\left\{b_{1}, c_{1}\right\}$. If $x y=b_{1} a_{2}$, let $X=\left(D^{*} \cup\left\{a_{2}\right\}\right) \backslash\left\{b_{2}, c_{2}\right\}$. If $x y=b_{1} b_{2}$, let $X=D^{*} \backslash\left\{c_{2}\right\}$. If $x y=b_{1} c_{2}$, let $X=D^{*} \backslash\left\{c_{1}\right\}$. Note that in all cases, $X$ is clearly a TD-set. To see that it is also locating, we observe that any vertex of $G_{i}, i \in[2]$, not in $X$ has a neighbor in $X \cap V\left(G_{i}\right)$ (to this end, also recall that $H_{1}$ and $H_{2}$ have no isolated vertices). Thus, if we had two vertices that are not located by $X$, we would have a 4-cycle in $G$, a contradiction. Hence, in each case the set $X$ is a LTD-set of $G$ of size $|D|-1=\frac{2}{3} n-1$, a contradiction. Therefore, $x=c_{1}$. Analogously, $y=c_{2}$. This is true for every edge $x y$ joining a vertex $x \in V_{1}$ and a vertex $y \in V_{2}$, implying that $G$ is the 2-corona of some connected nontrivial graph that contains no 4-cycles but contains a triangle. This completes the proof of Claim 8.B. (口)

By Claim 8.B, the graph $G$ contains no triangle, for otherwise the desired result follows. Hence, the girth of $G$ is at least 5 . Let $C: u_{0} u_{1} \ldots u_{k-1} u_{0}(k \geqslant 5)$ be a smallest cycle in $G$. Let $G^{\prime}=G-V(C)$. We build a subset $V_{1}$ of vertices of $G$ as follows (similarly to the proof of Claim 8.B). Let $V_{0}$ consist of $V(C)$ together with all vertices that belong to a component of $G^{\prime}$ isomorphic to $P_{1}, P_{2}$ or $P_{3}$. Since $G$ is twin-free and has girth at least 5 , 
we note that $G\left[V_{0}\right]$ is twin-free. Suppose that $S$ is a set of mutual twins of $G-V_{0}$. Since $G$ is twin-free, all but possibly one vertex in $S$ must be adjacent to a vertex of $C$. For each such set $S$ of mutual twins of $G-V_{0}$, we select $|S|-1$ vertices from $S$ that have a neighbor in $C$, and add these vertices to the set $V_{0}$ to form the set $V_{1}$ (possibly, $V_{1}=V_{0}$ ). Let $T=V_{1} \backslash V_{0}$. We note that since $G$ has girth at least 5 , the vertices in each set $S$ of mutual twins of $G-V_{0}$ are open twins, and have degree 1 in $G-V_{0}$ (if they were closed twins, they could not have a common neighbor since $G$ has girth at least 5 , but then they would form a $P_{2}$-component of $G^{\prime}$ ). Moreover they can have at most one neighbor in $V_{0}$, for otherwise they would have two or more neighbors in $V(C)$, but this would create a shorter cycle than $C$, contradicting its minimality. Hence, every vertex in $T$ has exactly one neighbor in $V_{0}$ (more precisely, in $V(C)$ ). Let $V_{2}=V(G) \backslash V_{1}$. Let $G_{1}=G\left[V_{1}\right]$ and if $V_{2} \neq \varnothing$, let $G_{2}=G\left[V_{2}\right]$.

Claim 8.C. If $G=G_{1}$, then $\gamma_{t}^{L}(G) \leqslant \frac{2}{3} n$. Further, $\gamma_{t}^{L}(G)=\frac{2}{3} n$ if and only if $G$ is isomorphic to a 6-cycle or is the 2-corona of the cycle $C$.

Proof of Claim 8.C. Suppose that $G=G_{1}$. If $T \neq \varnothing$, then this would imply that $V_{2} \neq \varnothing$, contradicting our supposition that $V(G)=V_{1}$. Hence, $T=\varnothing$, and so $V_{1}=V_{0}$. Thus, either $G$ is the $k$-cycle $C$ or $V(G) \neq V(C)$ and every component in $G^{\prime}=G-V(C)$ is isomorphic to $P_{1}, P_{2}$ or $P_{3}$. Suppose that $G=C$. Then, $n=k$. If $k=5$, then $G=C_{5}$ and $\gamma_{t}^{L}(G)=3<\frac{2}{3} n$. If $k=6$, then $G=C_{6}$ and $\gamma_{t}^{L}(G)=\frac{2}{3} n$. If $G=C$ and $k>6$, then, as observed in [16], $\gamma_{t}^{L}(G)=\gamma_{t}(G)=\lfloor n / 2\rfloor+\lceil n / 4\rceil-\lfloor n / 4\rfloor \leqslant \frac{1}{2} n+1<\frac{2}{3} n$. Hence we may assume that $G \neq C$, for otherwise the desired result follows. As observed earlier, every component of $G^{\prime}$ is isomorphic to $P_{1}, P_{2}$ or $P_{3}$. Among all components of $G^{\prime}$, let $P^{\prime}$ be chosen so that its order is maximum. We now consider the graph $F=G-V\left(P^{\prime}\right)$. Clearly, $F$ is twin-free, since $G$ is twin-free and removing $P^{\prime}$ from $G$ cannot create any twins. Applying the inductive hypothesis to the graph $F, \gamma_{t}^{L}(F) \leqslant \frac{2}{3}|V(F)|$. Further, $\gamma_{t}^{L}(F)=\frac{2}{3}|V(F)|$ if and only if $F$ is isomorphic to a 6-cycle, $C_{6}$, or is the 2-corona of some connected nontrivial graph that contains no 4-cycles.

Subclaim 8.C.1 If $\gamma_{t}^{L}(F)<\frac{2}{3}|V(F)|$, then the desired result of Claim 8.C holds.

Proof of Subclaim 8.C.1. Suppose that $\gamma_{t}^{L}(F)<\frac{2}{3}|V(F)|$. If $P^{\prime}=P_{3}$, consider a minimum LTD-set $D_{F}$ of $F$, and note that $D_{F}$ together with the two vertices of $P^{\prime}$ that have degree at least 2 in $G$, forms a LTD-set of $G$ of size strictly less that $\frac{2}{3} n$. Hence, we may assume that $P^{\prime}$ is isomorphic to $P_{1}$ or $P_{2}$. By our choice of $P^{\prime}$, this implies that every component of $G^{\prime}$ is isomorphic to $P_{1}$ or $P_{2}$. We now construct a set $Q$ with $V\left(P^{\prime}\right) \subset Q$. Renaming vertices of $C$, if necessary, we may assume that $u_{1}$ is the vertex of $C$ adjacent to a vertex of $P^{\prime}$. We initially define $Q$ to contain both $u_{1}$ and $u_{2}$, as well as all vertices that belong to a $P_{1^{-}}$or $P_{2}$-component of $G-\left\{u_{1}, u_{2}\right\}$. If $u_{3}$ has degree 2 in $G$ and $u_{4}$ has a leaf-neighbor in $G$, say $u_{4}^{\prime}$, then $u_{3}$ and $u_{4}^{\prime}$ are (open) twins in $G-Q$. In this case, we add the vertex $u_{3}$ to the set $Q$. Analogously, if $u_{0}$ has degree 2 in $G$ and $u_{k-1}$ has a leaf-neighbor in $G$, then we add the vertex $u_{0}$ to the set $Q$. By construction, the resulting graph $G-Q$ is twin-free, unless we have the special case when $k=5$, both $u_{0}$ and $u_{3}$ have degree 2 in $G$, and $u_{4}$ has degree 3 in $G$ with a leaf-neighbor in $G$. In this case, graph $G$ is determined 
and the set $\left\{u_{0}, u_{1}, u_{2}, u_{4}\right\}$ together with the vertices of every $P_{2}$-component in $G^{\prime}$ that have a neighbor in $V(C)$ forms a LTD-set of $G$ of size strictly less that $\frac{2}{3} n$. Hence, we may assume that the graph $F^{\prime}=G-Q$ is twin-free.

Applying the inductive hypothesis to the graph $F^{\prime}$ there exists a LTD-set, $D_{F}^{\prime}$, of $F^{\prime}$ of size at most $\frac{2}{3}\left|V\left(F^{\prime}\right)\right|$. Although $G[Q]$ is not necessarily twin-free, by similar arguments as before we can easily choose a set $D_{Q}$ of size at most $\frac{2}{3}|Q|$ such that $D_{F}^{\prime} \cup D_{Q}$ is a LTD-set of $G$ of size at most $\frac{2}{3} n$. Moreover, if $\left|D_{F}^{\prime} \cup D_{Q}\right|=\frac{2}{3} n$, then $F^{\prime}$ must be either the 2-corona of the path $G[V(C) \backslash Q]$, or $F^{\prime}=P_{6}$. Furthermore, $|Q|=6$ and $G[Q]$ is either a $P_{6}$, a $P_{4}$ with an additional leaf attached to each central vertex, or a $P_{5}$ with an additional leaf forming a twin with another leaf. If $F^{\prime}=P_{6}$ or $G[Q] \neq P_{6}$, we can readily find a LTD-set of $G$ strictly smaller than $\frac{2}{3} n$. Otherwise, $G$ is the 2-corona of $C$, and we are done. This completes the proof of Subclaim 8.C.1. (ㅁ)

By Subclaim 8.C.1, we may assume that $\gamma_{t}^{L}(F)=\frac{2}{3}|V(F)|$, for otherwise the desired result follows. If $F=C_{6}$, then $\gamma_{t}^{L}(G)<\frac{2}{3} n$, irrespective of whether $P^{\prime}$ is isomorphic to $P_{1}, P_{2}$ or $P_{3}$. Hence, we may assume that $F \neq C_{6}$, for otherwise the desired result follows. Thus, $F$ is the 2-corona of some connected nontrivial graph, say $F^{\prime}$, that contains no 4-cycles. Let $A_{F}$ and $B_{F}$ be the set of leaves and support vertices, respectively, in $F$, and let $C_{F}$ be the remaining vertices of $F$. Thus, $F^{\prime}=F\left[C_{F}\right]$. If $P^{\prime}$ is not isomorphic to $P_{3}$, or if $P^{\prime}$ is isomorphic to $P_{3}$ and contains a vertex adjacent to $A_{F}$ or $B_{F}$, then it is a simple exercise to see that $\gamma_{t}^{L}(G)<\frac{2}{3} n$. Further, if $P^{\prime}$ is isomorphic to $P_{3}$ and contains two or more vertices adjacent to vertices of $C_{F}$, then $\gamma_{t}^{L}(G)<\frac{2}{3} n$. If $P^{\prime}$ is isomorphic to $P_{3}$ and contains exactly one vertex adjacent to vertices of $C_{F}$, then $\gamma_{t}^{L}(G)=\frac{2}{3} n$ and $G$ is the 2 -corona of some connected nontrivial graph that contains no 4-cycles. This completes the proof of Claim 8.C. (口)

By Claim 8.C, we may assume that $G \neq G_{1}$, i.e., $V_{2} \neq \varnothing$. An identical proof as in the proof of Subclaim 8.B.1 shows that $G_{2}$ is twin-free. Let $D_{2}$ be a minimum LTD-set of $G_{2}$. Applying the induction hypothesis to each component of $G_{2}$, the set $D_{2}$ satisfies $\left|D_{2}\right| \leqslant \frac{2}{3}\left|V_{2}\right|$. Further, if $\left|D_{2}\right|=\frac{2}{3}\left|V_{2}\right|$, then each component of $G_{2}$ is isomorphic to a 6 -cycle or is the 2-corona of some connected nontrivial graph that contains no 4-cycles.

Recall that $G\left[V_{0}\right]$ is twin-free. We now build sets $V_{1}^{\prime}$ and $T^{\prime}$ such that $V_{0} \subseteq V_{1}^{\prime} \subseteq$ $V_{1}=V_{0} \cup T$ and $T^{\prime} \subseteq T$, as follows. Initially, we let $V_{1}^{\prime}=V_{0}$ and $T^{\prime}=T$. We consider the vertices of $T$ sequentially. Let $t$ be a vertex in $T$, and recall that $t$ has exactly one neighbor, say $u_{t}$, in $V_{0}$, and such a neighbor belongs to $V(C)$. If $u_{t}$ has no leaf-neighbor in $G\left[V_{1}^{\prime}\right]$, we add $t$ to $V_{1}^{\prime}$ and remove $t$ from $T^{\prime}$. We iterate this process until all vertices of $T$ have been considered. Let $G_{1}^{\prime}$ be the resulting graph $G\left[V_{1}^{\prime}\right]$. This process yields a new partition of $V(G)$ into sets $V_{2}, V_{1}^{\prime}$ and $T^{\prime}$. Since $G\left[V_{0}\right]$ is twin-free, by construction of the set $V_{1}^{\prime}$, the graph $G_{1}^{\prime}$ is also twin-free. Since $V_{2} \neq \varnothing$, the order of $G_{1}^{\prime}$ is less than $n$ and we can therefore apply the induction hypothesis to the connected twin-free graph $G_{1}^{\prime}$. Let $D_{1}^{\prime}$ be a minimum LTD-set of $G_{1}^{\prime}$. By the induction hypothesis, the set $D_{1}^{\prime}$ satisfies $\left|D_{1}^{\prime}\right| \leqslant \frac{2}{3}\left|V_{1}^{\prime}\right| \leqslant \frac{2}{3}\left|V_{1}\right|$. Further, if $\left|D_{1}^{\prime}\right|=\frac{2}{3}\left|V_{1}^{\prime}\right|$, then $G_{1}^{\prime}$ is isomorphic to a 6 -cycle or is the 2-corona of some connected nontrivial graph that contains no 4-cycles.

We claim that $D=D_{1}^{\prime} \cup D_{2}$ is a LTD-set of $G$. By the construction of the set $T^{\prime}$, for 
each vertex $t$ of $T^{\prime}$, there is a twin, say $t^{\prime}$, of $t$ in $G-V_{0}$ that belongs to $V_{2}$ and has degree 1 in $G_{2}$. The common neighbor of $t$ and $t^{\prime}$ in $V_{2}$ must belong to $D_{2}$. Further, since $t$ has not been removed from $T^{\prime}$ during the construction of $T^{\prime}$, the vertex $t$ has a neighbor $u_{t}$ in $V(C)$ which has a leaf-neighbor in $G_{1}^{\prime}$, implying that the vertex $u_{t}$ belongs to $D_{1}^{\prime}$. Hence, $t$ is dominated by two vertices of $D_{1}^{\prime} \cup D_{2}$ and is therefore located by $D$, for otherwise we would have a 4-cycle in $G$. Thus, every vertex of $T^{\prime}$ is located by $D$. Since $D_{1}^{\prime}$ and $D_{2}$ are TD-sets of $G_{1}^{\prime}$ and $G_{2}$, respectively, and since every vertex in $T^{\prime}$ is dominated by $D$, the set $D$ is a TD-set of $G$. Suppose, to the contrary, that $D$ is not locating. Then there is a pair of vertices, $u$ and $v$, that is not located by $D$. As observed earlier, neither $u$ nor $v$ belong to $T^{\prime}$. Since $D_{2}$ is locating in $G_{2}$, we note that $(u, v) \notin V_{2} \times V_{2}$. Analogously, since $D_{1}^{\prime}$ is locating in $G_{1}^{\prime}$, we note that $(u, v) \notin V_{1}^{\prime} \times V_{1}^{\prime}$. If $(u, v) \in V_{1}^{\prime} \times V_{2}$, then $u$ is dominated by a vertex of $D_{1}^{\prime}$ and $v$ is dominated by a vertex of $D_{2}$. Hence, $u$ and $v$ must both be dominated by these two vertices. But then these four vertices would form a 4 -cycle, a contradiction. Hence, $(u, v) \notin V_{1}^{\prime} \times V_{2}$. Analogously, $(u, v) \notin V_{2} \times V_{1}^{\prime}$. This contradicts our supposition that $u$ and $v$ are not located by $D$. Therefore, $D$ is a LTD-set of $G$, and so

$$
\gamma_{t}^{L}(G) \leqslant|D|=\left|D_{1}^{\prime}\right|+\left|D_{2}\right| \leqslant \frac{2}{3}\left|V_{1}^{\prime}\right|+\frac{2}{3}\left|V_{2}\right| \leqslant \frac{2}{3}\left|V_{1}\right|+\frac{2}{3}\left|V_{2}\right|=\frac{2}{3} n .
$$

This establishes the desired upper bound. Suppose next that $\gamma_{t}^{L}(G)=\frac{2}{3} n$. Then we must have equality throughout the Inequality Chain (3). In particular, $\left|D_{1}^{\prime}\right|=\frac{2}{3}\left|V_{1}^{\prime}\right|=$ $\frac{2}{3}\left|V_{1}\right|$ and $\left|D_{2}\right|=\frac{2}{3}\left|V_{2}\right|$. This in turn implies that $T^{\prime}=\varnothing$. Using an analogous proof as in the proof when equality holds in the Inequality Chain (2) in the proof of Claim 8.B, the graph $G$ can be shown to be the 2-corona of some connected nontrivial graph that contains no 4-cycles. Since the proof is very similar, we omit the details. This completes the proof of Theorem 8 .

\section{Graphs with given minimum degree}

We now discuss the special case of graphs of given minimum degree.

\subsection{Minimum degree two}

If we forbid a certain set of six graphs (each of them of order at most 10), then it is known (see [17]) that every connected graph $G$ of order $n$ with $\delta(G) \geqslant 2$ satisfies $\gamma_{t}(G) \leqslant 4 n / 7$. However, for graphs with minimum degree 2, the location-total domination number can be much larger than the total domination number. For example, let $G$ be the graph obtained by taking the disjoint union of $k \geqslant 25$-cycles, adding a new vertex $v$ and joining $v$ with an edge to exactly one vertex from each 5 -cycle. The resulting twin-free graph $G$ has order $n=5 k+1$, minimum degree $\delta(G)=2$ and satisfies $\gamma_{t}^{L}(G)=3 k=\frac{3}{5}(n-1)$ and $\gamma_{t}(G)=2(k+1)=\frac{2}{5}(n-1)+2$.

We believe that Conjecture 1 can be strengthened for graphs with minimum degree at least 2 and pose the following question. 
Question 9. Is it true that every twin-free graph with order $n$, no isolated vertices and minimum degree 2 satisfies $\gamma_{t}^{L}(G) \leqslant \frac{3 n}{5}$ ?

If Question 9 is true, then the bound is asymptotically tight by the examples given earlier.

\subsection{Large minimum degree}

The following is an upper bound on $\gamma_{t}(G)$ according to the minimum degree $\delta$ of $G$.

Theorem 10 (Henning,Yeo [20]). If $G$ is a graph with minimum degree $\delta \geqslant 1$ and order $n$, then

$$
\gamma_{t}(G) \leqslant\left(\frac{1+\ln \delta}{\delta}\right) n
$$

Using Observation 6, we obtain the following corollary of the results in $[12,13]$ and Theorem 10.

Corollary 11. Let $G$ be a twin-free graph of minimum degree $\delta \geqslant 1$. We have

$$
\gamma_{t}^{L}(G) \leqslant\left(\frac{2}{3}+\frac{1+\ln \delta}{\delta}\right) n .
$$

Moreover, if $G$ is a bipartite, co-bipartite or split graph, then

$$
\gamma_{t}^{L}(G) \leqslant\left(\frac{1}{2}+\frac{1+\ln \delta}{\delta}\right) n
$$

If Conjecture 3 holds, we always have $\gamma_{t}^{L}(G) \leqslant\left(\frac{1}{2}+\frac{1+\ln \delta}{\delta}\right) n$.

It follows from Corollary 11 that Conjecture 1 asymptotically holds for large minimum degree, in the sense that $\lim _{\delta \rightarrow \infty}\left(\frac{2}{3}+\frac{1+\ln \delta}{\delta}\right)=\frac{2}{3}$. Moreover, Conjecture 1 holds for bipartite, co-bipartite, and split graphs with minimum degree $\delta \geqslant 26$. Finally, if Conjecture 3 holds, then Conjecture 1 holds whenever $\delta \geqslant 26$.

\section{Conclusion}

A classic result in total domination theory in graphs is that every connected graph of order $n \geqslant 3$ has a total dominating set of size at most $\frac{2}{3} n$. In this paper, we conjecture that every twin-free graph of order $n$ with no isolated vertex has a locating-total dominating set of size at most $\frac{2}{3} n$ and we prove our conjecture for graphs with no 4 -cycles. We also prove that our conjecture, namely Conjecture 1, holds asymptotically for large minimum degree. Since Conjecture 3 was proved for bipartite graphs [13] and cubic graphs [11], can we prove Conjecture 1 for these classes as well? 


\section{References}

[1] M. Blidia, M. Chellali, F. Maffray, J. Moncel, and A. Semri. Locating-domination and identifying codes in trees, Australas. J. Combin. 39 (2007), 219-232.

[2] M. Blidia and W. Dali. A characterization of locating-total domination edge critical graphs, Discuss. Math. Graph Theory 31(1) (2011), 197-202.

[3] M. Blidia, O. Favaron, and R. Lounes. Locating-domination, 2-domination and independence in trees, Australas. J. Combin. 42 (2008), 309-319.

[4] R. C. Brigham, J. R. Carrington, and R. P. Vitray. Connected graphs with maximum total domination number. J. Combin. Comput. Combin. Math. 34 (2000), 81-96.

[5] M. Chellali. On locating and differentiating-total domination in trees, Discuss. Math. Graph Theory 28(3) (2008), 383-392.

[6] M. Chellali and N. Jafari Rad. Locating-total domination critical graphs, Australas. J. Combin. 45 (2009), 227-234.

[7] X.G. Chen and M.Y. Sohn. Bounds on the locating-total domination number of a tree, Discrete Appl. Math. 159 (2011), 769-773.

[8] E. J. Cockayne, R. M. Dawes, and S. T. Hedetniemi. Total domination in graphs. Networks 10 (1980), 211-219.

[9] C. J. Colbourn, P. J. Slater, and L. K. Stewart. Locating-dominating sets in seriesparallel networks. Congr. Numer. 56 (1987), 135-162.

[10] A. Finbow and B. L. Hartnell. On locating dominating sets and well-covered graphs. Congr. Numer. 65 (1988), 191-200.

[11] F. Foucaud and M. A. Henning. Location-domination and matching in cubic graphs. Discrete Math. 339 (2016), 1221-1231.

[12] F. Foucaud, M. A. Henning, C. Löwenstein, and T. Sasse. Locating-dominating sets in twin-free graphs. Discrete Appl. Math. 200 (2016), 52-58.

[13] D. Garijo, A. González, and A. Márquez. The difference between the metric dimension and the determining number of a graph. Appl. Math. Comput. 249 (2014), 487-501.

[14] T. W. Haynes, S. T. Hedetniemi, and P. J. Slater, Fundamentals of Domination in Graphs, Marcel Dekker, Inc. New York, 1998.

[15] T. W. Haynes, S. T. Hedetniemi, and P. J. Slater (eds), Domination in Graphs: Advanced Topics, Marcel Dekker, Inc. New York, 1998.

[16] T. W. Haynes, M. A. Henning, and J. Howard. Locating and total dominating sets in trees. Discrete Appl. Math. 154 (2006), 1293-1300.

[17] M. A. Henning. Graphs with large total domination number. J. Graph Theory 35(1) (2000), 21-45.

[18] M. A. Henning and C. Löwenstein. Locating-total domination in claw-free cubic graphs. Discrete Math. 312(21) (2012), 3107-3116. 
[19] M. A. Henning and N. J. Rad. Locating-total domination in graphs, Discrete Appl. Math. 160 (2012), 1986-1993.

[20] M. A. Henning and A. Yeo. A transition from total domination in graphs to transversals in hypergraphs. Quaestiones Math. 30 (2007), 417-436.

[21] M. A. Henning and A. Yeo. Total domination in graphs, Springer-Verlag, 2013.

[22] O. Ore. Theory of graphs. Amer. Math. Soc. Transl. 38 (Amer. Math. Soc., Providence, RI, 1962), 206-212.

[23] D. F. Rall and P. J. Slater. On location-domination numbers for certain classes of graphs. Congr. Numer. 45 (1984), 97-106.

[24] P. J. Slater. Dominating and location in acyclic graphs. Networks 17 (1987), 55-64.

[25] P. J. Slater. Dominating and reference sets in graphs. J. Math. Phys. Sci. 22 (1988), 445-455.

[26] P. J. Slater. Locating dominating sets and locating-dominating sets. In Y. Alavi and A. Schwenk, editors, Graph Theory, Combinatorics, and Applications, Proc. Seventh Quad. Internat. Conf. on the Theory and Applications of Graphs, pages 1073-1079. John Wiley \& Sons, Inc., 1995. 